\title{
Estimating the solar wind conditions during an extreme geomagnetic storm: a case study of the event that occurred on March 13-14, 1989
}

Tsutomu Nagatsuma ${ }^{1 *}$, Ryuho Kataoka ${ }^{2,3}$ and Manabu Kunitake

\begin{abstract}
The solar wind conditions of an extreme geomagnetic storm were examined using magnetic field observations obtained from geosynchronous satellites and the disturbance storm-time (Dst) index. During geosynchronous magnetopause crossings (GMCs), magnetic field variations at the magnetosheath, which is the modulated interplanetary magnetic field (IMF), were observed by geosynchronous satellite. The dawn to dusk solar wind electric field $\left(\mathrm{VB}_{\mathrm{S}}\right.$ ) was estimated from the Dst index by using an empirical formula for Dst prediction; these data were then used to estimate the IMF and solar wind speed. This method was applied in the analysis of an extreme geomagnetic storm event that occurred on March 13-14, 1989, for which no direct solar wind information was available. A long duration of the GMC was observed after the second storm sudden commencement (SSC) of this event. The solar flare possibly associated with the second SSC of this storm event was identified as the March $12 \mathrm{M7.3/2B}$ flare. The IMF $B_{z}$ was estimated to be about $-50 \mathrm{nT}$ with a solar wind speed of about $960 \mathrm{~km} / \mathrm{s}$ during the $5 \mathrm{~h}$ in which the main phase of the storm rapidly developed, assuming an Alfvén Mach number $\left(M_{A}\right)$ during this period of more than 2.
\end{abstract}

Keywords: Extreme geomagnetic storm; Geosynchronous magnetopause crossing; Geomagnetic indices; Solar wind parameters

\section{Background}

Geomagnetic disturbances are driven by the effect of the solar wind-magnetosphere-ionosphere compound system. A number of studies have been conducted to obtain empirical relationships for the compound system and to develop a numerical simulation code for understanding this interaction (e.g., Tanaka 2007 and references therein). However, there is little knowledge of this compound system under extreme conditions because of the infrequent occurrence of extreme events.

To examine geomagnetic disturbances under extreme conditions, the following two aspects are important. The first is the condition of the solar wind, which is the main driver of geomagnetic disturbances, and the second is the behavior of the solar wind-magnetosphere-ionosphere coupling processes under extreme conditions of solar wind. Because of the complex nature of this system, it can

\footnotetext{
* Correspondence: tnagatsu@nict.go.jp

'National Institute of Information and Communications Technology, Tokyo, Japan

Full list of author information is available at the end of the article
}

be difficult to assess whether the response of the system during a normal space environment can be applied to extreme conditions of solar wind. Thus, the study of events occurring under extreme conditions is important for understanding the behavior of the compound system.

Coverage of solar wind observation was limited prior to operation of the Advanced Composition Explorer (ACE) at the L1 point in 1997. During the maximum period of solar cycle 22, almost the only solar wind monitoring platform was Interplanetary Monitoring Platform (IMP) 8, which had a 35 Earth radii circular orbit, and was used to measure Earth's magnetotail, magnetosheath, and solar wind. The coverage of solar wind observation by IMP 8 was only for 7 to 8 days of every 12.5 days, which was the orbital period of IMP 8 . Such circumstances led to many severe geomagnetic disturbances for which there was no solar wind observation in the maximum period of solar cycle 22. Many severe geomagnetic storm events have thus not been analyzed in detail owing to a lack of solar wind information. For example, an extreme storm that occurred on March 13-14, 
1989, which is the focus of this paper, had no solar wind coverage during its onset, main phase, and most of the recovery phase. Therefore, McKenna-Lawlor et al. (2005) attempted to reconstruct the interplanetary shocks from five major flares hitting both Earth and Mars during the March 9-23, 1989, by using the HakamadaAkasofu-Fry version 2 (HAFv.2) solar wind model (Fry et al. 2001, 2003). Other papers have reconstructed solar wind parameters during large storms by using geomagnetic variations or indices (e.g., Li et al. 2006; Cliver et al. 2009).

The three major solar wind monitoring platforms currently around Earth's orbit include ACE and Solar Terrestrial Relations Observatories (STEREO) Ahead (A) and Behind (B). STEREO-A and STEREO-B are drifting in heliocentric orbits away from Earth; the former is leading, and the latter is lagging. Baker et al. (2013) examined the extreme solar wind conditions obtained from STEREO-A on July 23-24, 2012. They also used the WSA-ENLIL model to estimate the proton density of the solar wind for this event because the quality of the low-energy particle measurements might have been poor owing to contamination from high-energy particles. On the basis of their data analysis, they suggested that an extreme solar wind with their estimated conditions could produce an extreme geomagnetic storm if it reached the geospace environment; the lowest possible disturbance storm-time (Dst) index was $-1182 \mathrm{nT}$.

In this paper, we estimate the solar wind conditions for the extreme storm event that occurred on March $13-14,1989$, by using the geostationary magnetopause crossing (GMC) period obtained from the Geostationary Operational Environmental Satellites (GOES) magnetic field data and the Dst index. The solar origin of this event is also discussed.

\section{Overview of the March 13-14, 1989, storm event}

The geomagnetic storm that occurred on March 13-14, 1989, was the most extreme geospace disturbance since 1957. The minimum value of the Dst index during this event was $-589 \mathrm{nT}$ at $02 \mathrm{UT}$ on March 14. From a probabilistic viewpoint, an event of this scale may occur once every 60 years (Kataoka 2013). Two storm sudden commencements (SSCs) have been identified for this storm event at 0128 and 0747 UT, both on
March 13 (e.g., Fujii et al. 1992), with amplitudes of 43 and $76 \mathrm{nT}$ at Kakioka Observatory, respectively. The main phase of the storm rapidly developed after 20 UT on March 13. The magnitude of the Dst index decreased by $330 \mathrm{nT}$ in $5 \mathrm{~h}$ and reached a minimum of $-589 \mathrm{nT}$.

This event is also well known for its practical implications. The Hydro-Quebec power system collapsed because of the strong geomagnetically induced current (GIC) that flowed in power lines in North America. As a result, six million residents in the province of Quebec were left without electrical power for more than $9 \mathrm{~h}$. Furthermore, a low-latitude aurora was visible across the southern United States during the night of March 13 and early hours of March 14 (Allen et al. 1989). Although the solar wind information is not available for this period, geospace disturbances during this extreme storm have been studied by using satellite data (e.g., Fujii et al. 1992; Greenspan et al. 1991; Okada et al. 1993; Rasmussen and Greenspan 1993; Rich and Denig 1992; Shinbori et al. 2005; Sojka et al. 1994) and ground-based ionospheric data (e.g., Batista et al. 1991; Hajkowicz 1991; Lakshmi et al. 1991; Walker and Wong 1993; Yeh et al. 1992).

On the contrary, the solar activity possibly related to this extreme geomagnetic storm was less extreme. Table 1 shows flare events and related phenomena extracted from the event list of solar flares in the solar geophysical data issued by National Geophysical Data Center/National Oceanic and Atmospheric Administration (NGDC/NOAA) and from the coronal mass ejection (CME) catalog based on Solar Maximum Mission (SMM) coronagraph observations (Burkepile and St. Cyr 1993). These events may be related to the storm that occurred on March 13-14. During this period, solar X-ray flares of intensity greater than M5.0 occurred only from active region 5395. From March 6 to March 18, many solar flares and CMEs associated with this large and complex active region occurred (Feynman and Hundhausen 1994). The most intense X-ray flare from this active region was X15 at 1350 UT on March 6. In contrast, the intensities of the major X-ray flares shown in Table 1 were less than that of X5. The X-ray intensity and duration of these events are almost comparable except for the X4.5/3B flare that occurred on March 10.

The shock transit speed of this event was also not extreme. According to Cliver et al. (1990), the solar flare associated with the first SSC of this storm event was

Table 1 List of optical and X-ray solar flares with X-ray intensities of more than M5.0

\begin{tabular}{lllllllllll}
\hline Date & Start & Max & End & Lat. & CMD & Opt & X-ray & NOAA\# & CME & Radio burst \\
\hline $3 / 10$ & 1848E & 1912U & 2158 & N31 & E22 & 3B & X4.5 & 5395 & Yes & Type II, IV \\
$3 / 11$ & 0829E & 0905 & 0944D & N29 & E16 & 2B & M9.7 & 5395 & & \\
$3 / 11$ & $1535 E$ & 1538 & 1601D & N28 & E13 & 2B & X1.2 & 5395 & Y \\
$3 / 11$ & 1933E & 1940 & 2016D & N27 & E10 & 2B & X1.3 & 5395 & Yes & Type IV \\
$3 / 12$ & 0016E & 0029 & 0043D & N28 & E09 & 2B & M7.3 & 5395 & \\
\hline
\end{tabular}


identified as the March $10 \mathrm{X} 4.5 / 3 \mathrm{~B}$ flare, which is in the top row of Table 1. McKenna-Lawlor et al. (2005) also calculated an interplanetary shock propagation by using the HAFv2 model with the solar origin of the X4 flare on March 10 and the first SSC of the storm on March 13-14. The transit time from the occurrence of the solar flare to the first SSC in this event has been estimated to be $54.8 \mathrm{~h}$, which is 3.8 times longer than the event on that occurred on August 4, 1972, with a transit time of $14.6 \mathrm{~h}$ (Cliver et al. 1990). The maximum solar wind speed of the interplanetary shock that produced the first SSC of this event was estimated to be about $550 \mathrm{~km} / \mathrm{s}$ in their paper. However, they did not discuss the second SSC of this event and its solar origin. At the time of the second SSC, the Dst had dropped to $\sim-130 \mathrm{nT}$. The rapid enhancement of the ring current started at about 20 UT on March 13, approximately $12 \mathrm{~h}$ after the second SSC. The existence of the two SSCs strongly suggests that this great geomagnetic storm was caused by the combination of two CMEs (solar flares).

\section{Methods}

Estimating solar wind conditions during the March 13-14, 1989, event

In this study, we focus on the GMC by GOES. Because of the compression due to the high dynamic pressure of the solar wind and the erosion due to the enhanced dayside reconnection based on the intense southward interplanetary magnetic field (IMF), the location of the dayside magnetopause shrank and sometimes reached inside the geosynchronous orbit (6.6 Earth radii).

Figure 1 shows the magnetic field variation observed by GOES 06 and 07 represented by red and blue lines, respectively. The variations of magnetic field intensity, $X, Y$, and $Z$ components of the magnetic field variations based on the geocentric solar magnetospheric (GSM) coordinate system and the variations of the Dst index are plotted from top to bottom in the figure. Two dotted vertical lines indicate times of the first and the second SSC, respectively. In this period, the geographic longitude and local noon of GOES 06 and 07 were $135.5^{\circ}$ (2102 UT) and $108.5^{\circ}$ (1814 UT), respectively.

During the period from 14 UT on March 13 to 01 UT on March 14, several negative excursions of the GSM-Z magnetic field component were recorded for both GOES 06 and 07, which is a manifestation of the GMC. The extreme duration of the GMC in this event should be noted here. Excluding the time period from 1735 to 1945 UT, the GMC is continued for almost $11 \mathrm{~h}$, which means that the GMC occurred during almost the entire daytime period of the GOES satellites.

Another important point to note here is that both the GSM- $Y$ and GSM- $Z$ components of the magnetic field variations from GOES 06 and 07 were almost coherent during the GMC. This characteristic suggests that both GOES 06 and 07 simultaneously observed the magnetosheath field during the GMC, which was the IMF modulated by the bow shock. Therefore, it should be possible to estimate the variations of the IMF from the magnetic field observations of GOES 06 and 07 during the GMC in the same manner as that of a solar wind monitoring satellite.

By analyzing the data of the storm that occurred on November 20-21, 2003, which is the fifth largest storm since 1957, Nagatsuma et al. (2007) showed that the GSM- $Y$ and GSM- $Z$ components of the magnetic field variations from GOES during the GMC were four times larger than the IMF assuming that the Alfvén Mach number $\left(M_{\mathrm{A}}\right)$ of the solar wind was more than 2 . Thus, the GSM- $Y$ and GSM- $Z$ components of the IMF were estimated to be a quarter of the magnetic field variations observed by GOES 06 and 07 during the GMC when $M_{\mathrm{A}}$ for the solar wind was more than 2 .

The other important point is that the GMC occurred because of the high dynamic pressure of the solar wind and the erosion effect of the intense southward IMF (e.g., Rufanach et al. 1989). This means that the occurrence of the GMC also provides information about the dynamic pressure condition of the solar wind in this event. By using the empirical model of the magnetopause, the lower limit of the solar wind dynamic pressure during the GMC can be estimated. In this study, the magnetopause model developed by Shue et al. $(1998,2000)$ was used for our estimation. The equations in their model are

$$
\begin{aligned}
& r_{0}=\left\{10.22+1.29 \tanh \left[0.184\left(B_{\mathrm{z}}+8.14\right)\right]\right\}\left(D_{\mathrm{p}}\right)^{-1 / 6.6}, \\
& \alpha=\left(0.58-0.007 B_{\mathrm{z}}\right)\left[1+0.024 \ln \left(D_{\mathrm{p}}\right)\right],
\end{aligned}
$$

where $r_{0}, \alpha, D_{\mathrm{p}}$, and $B_{\mathrm{z}}$ represent the standoff distance at the subsolar point, the level of tail flaring, the solar wind dynamic pressure, and north-south component of the IMF, respectively. To estimate $D_{\mathrm{p}}$ from these equations, the IMF $B_{\mathrm{z}}$ estimated from the GOES observations is used as an input parameter. The estimation of $D_{\mathrm{p}}$ from this empirical model is limited to the time at which the solar zenith angle of the satellite is less than $60^{\circ}$ because this model should be applied only around the noon sector.

Another way to estimate the solar wind parameters is to use the geomagnetic index, which is a manifestation of the solar wind-magnetosphere-ionosphere coupling. By using an empirical model of geomagnetic indices, Nagatsuma et al. (2007) estimated the $\mathrm{VB}_{\mathrm{s}}$ and the merging electric field $\left(E_{\mathrm{m}}\right)$ from the Dst and the PC index for the Northern Hemisphere (PCN) during the storm that 


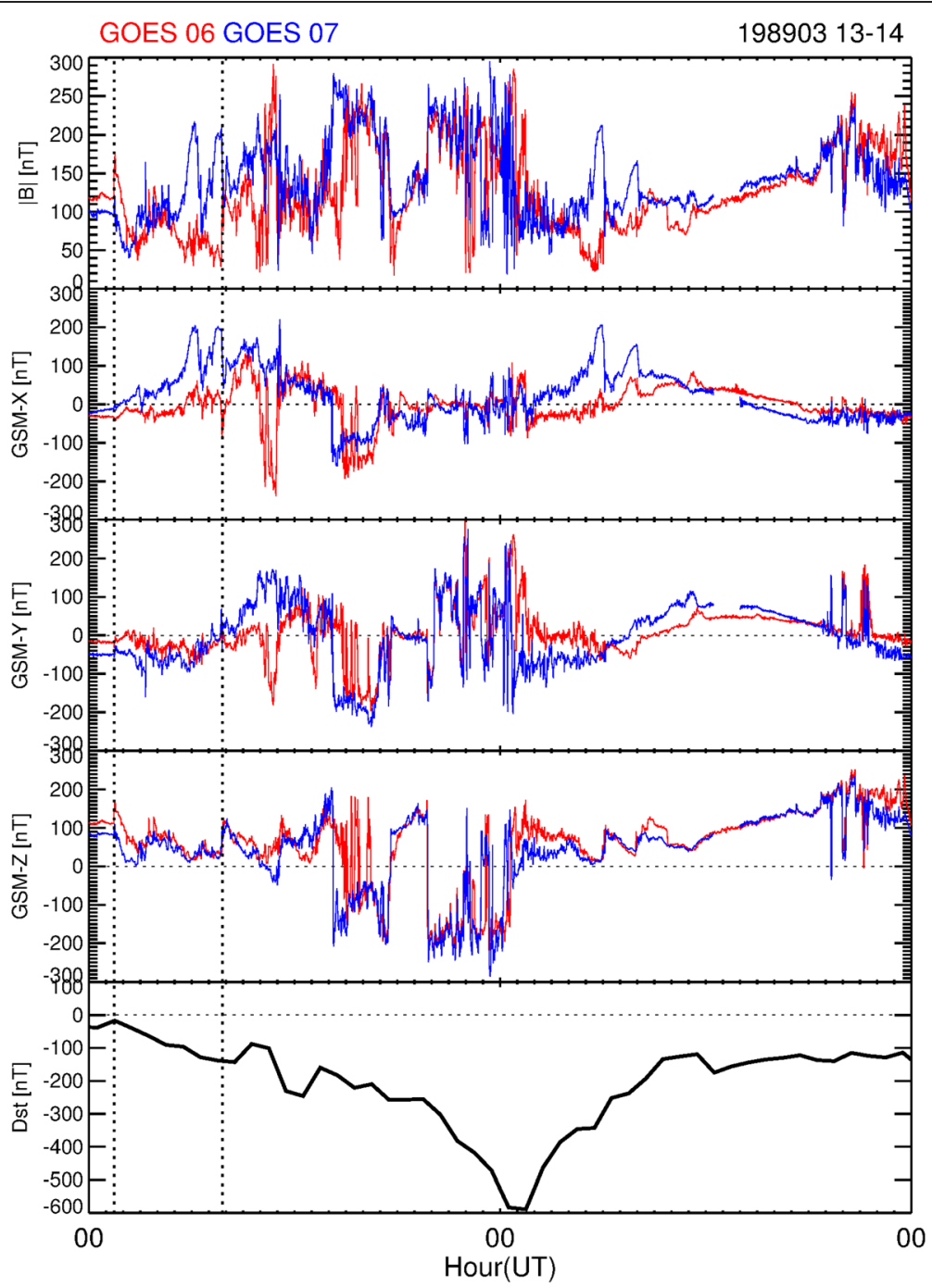

Fig. 1 Magnetic field variations at geosynchronous orbit on March 13-14, 1989, observed by Geostationary Operational Environmental Satellites (GOES) 06 and 07 are represented by red and blue lines, respectively. The magnetic field intensity, geocentric solar magnetospheric (GSM)-X, GSM-Y, and GSM-Z components of the magnetic field variations, and the variation of the Dst index are plotted from top to bottom. Two dotted vertical lines indicate the first and the second storm sudden commencement (SSC), respectively

occurred on November 20-21, 2003, respectively. They also showed that these estimations are in good agreement with values estimated from satellite observations of the solar wind. We applied the same method of $\mathrm{VB}_{\mathrm{s}}$ estimation to this event.

The solar wind parameter $\mathrm{VB}_{\mathrm{s}}$ was estimated from the following equations obtained by O'Brien and McPherron (2000):

$$
\begin{aligned}
& Q(t)=-4.4\left(\mathrm{VB}_{\mathrm{s}}-0.50\right) \\
& Q(t)=\mathrm{dDst}^{*} / \mathrm{dt}+\mathrm{Dst}^{*} / \tau, \\
& \tau=2.4 \exp \left[9.74 /\left(4.69+\mathrm{VB}_{\mathrm{s}}\right)\right] .
\end{aligned}
$$

Here $Q(t)$ is the injection rate of the solar wind energy for the development of the ring current, and $\tau$ is the decay time. Dst: is the pressure-corrected Dst index obtained from the following equation:

$$
\mathrm{Dst}^{*}=\text { Dst }-7.26 \sqrt{D_{\mathrm{p}}}+11 .
$$

If the dynamic pressure correction is negligible, the Dst index is used as the Dst" index. By using Eqs. (3), (4), and (5), we estimated the $\mathrm{VB}_{\mathrm{s}}$ from the Dst index.

The estimated solar wind parameters for the GMC period of the GOES data and the Dst index are shown in Fig. 2. The estimated GSM- $Y$ and GSM- $Z$ components of the IMF and the lower limit of the solar wind dynamic pressure are plotted from top to the third panel. The $\mathrm{VB}_{\mathrm{s}}$ is plotted in the fourth panel. A solid line represents the $\mathrm{VB}_{\mathrm{s}}$ estimated from the difference of the Dst index $(\Delta \mathrm{Dst})$, 


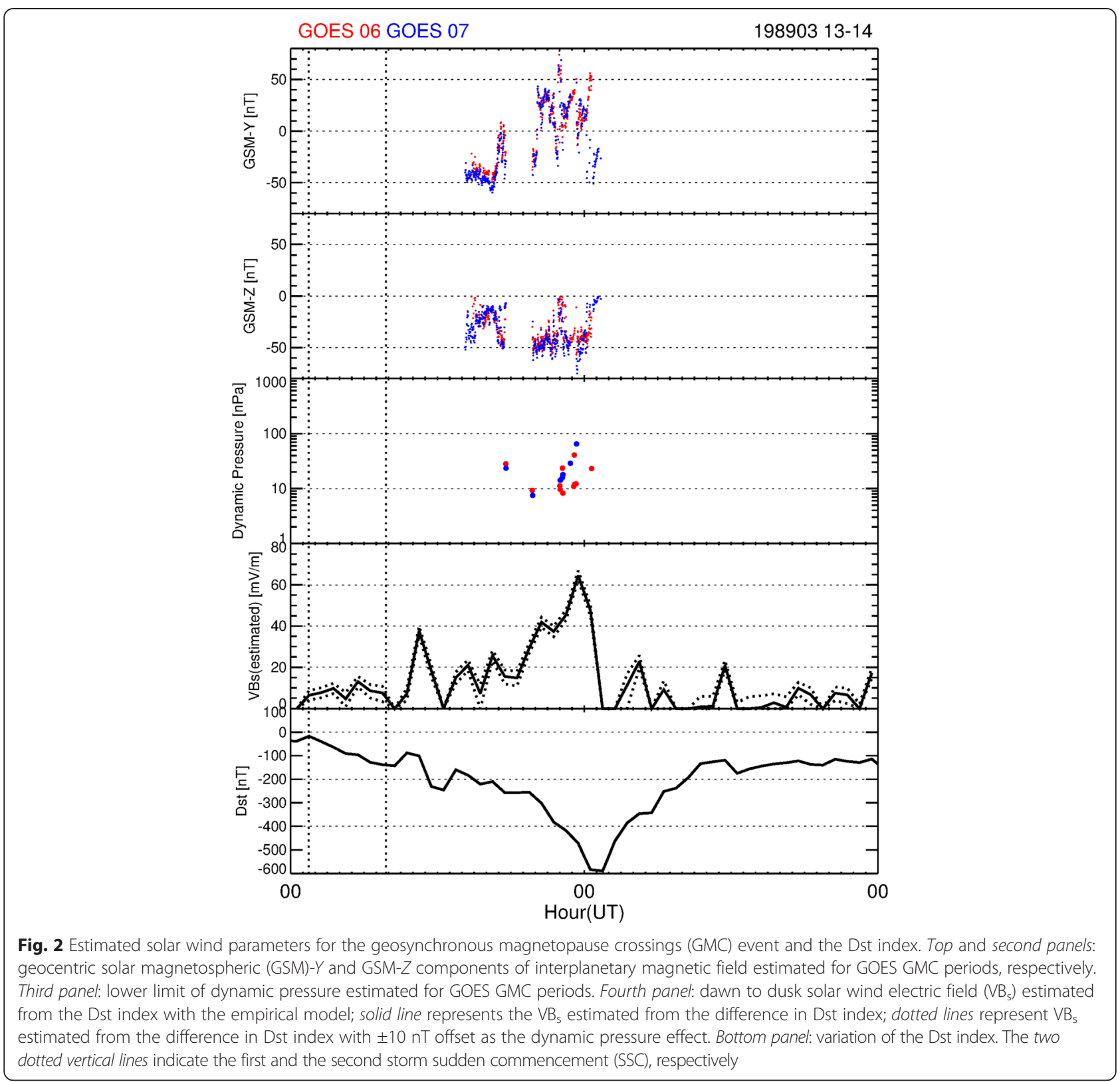

and dotted lines represent the $\mathrm{VB}_{\mathrm{s}}$ estimated from $\Delta \mathrm{Dst}$ with $\pm 10 \mathrm{nT}$ offset as the dynamic pressure effect. The offset of the Dst index due to dynamic pressure enhancement was about $10 \mathrm{nT}$, when the 1-h averaged solar wind dynamic pressure changed from 10 to $20 \mathrm{nPa}$. The Dst index is plotted in the bottom panel of the figure. It is apparent that the interruption of the GMC between 1735 and 1945 UT is in good agreement with the time during which the Dst index was stable. The average magnitude of the southward IMF $B_{\mathrm{z}}$ was $-30 \mathrm{nT}$ $(-50 \mathrm{nT})$ for the former (latter) part of GMC period. The lower limit of the dynamic pressure of the solar wind was about 10 to $80 \mathrm{nPa}$. $\mathrm{VB}_{\mathrm{s}}$ was about $15 \mathrm{mV} / \mathrm{m}$ and $40-60 \mathrm{mV} / \mathrm{m}$ during the first and latter parts, respectively. It is apparent that the dynamic pressure effect of Dst is negligible because of the small difference of the dynamic pressure effect.

Next, we estimated the solar wind speed $\left(V_{\mathrm{sw}}\right)$ from the estimated IMF $B_{\mathrm{z}}$ and $\mathrm{VB}_{\mathrm{s}}$ averaged over $1 \mathrm{~h}$. The estimated solar wind speed is highly scattered because this estimation is rather sensitive to the magnitude of the IMF $B_{\mathrm{z}}$. The average solar wind flow speed during this period was $960 \mathrm{~km} / \mathrm{s}$.

\section{Results and discussion}

On the basis of the estimated solar wind speed in the previous section, we attempted to identify the solar origin 
Table 2 List of X-ray solar flares of more than M5.0 and estimated shock speed and maximum solar wind speed

\begin{tabular}{llllll}
\hline Day & Start & X-ray & $\begin{array}{l}V_{\text {sh }}(\mathrm{second} \\
\text { SSC) }(\mathrm{km} / \mathrm{s})\end{array}$ & $\begin{array}{l}V_{\max }(\mathrm{second} \mathrm{SSC})(\mathrm{km} / \mathrm{s}) \\
\text { Cliver et al. 1990 }\end{array}$ & $\begin{array}{l}V_{\max }(\mathrm{second} \mathrm{SSC})(\mathrm{km} / \mathrm{s}) \\
\text { Belov et al. 2008 }\end{array}$ \\
\hline 10 & $1848 \mathrm{E}$ & X4.5 & 680 & 490 & $547-600$ \\
11 & 0829E & M9.7 & 880 & 640 & $645-710$ \\
11 & 1535E & X1.2 & 1040 & 760 & $724-798$ \\
11 & 1933E & X1.3 & 1150 & 850 & $778-859$ \\
12 & 0016E & M7.3 & 1320 & 980 & $906-952$ \\
\hline
\end{tabular}

of the second SSC and the development of the main phase of this extreme storm event. The maximum solar wind flow speed can be estimated from the transit speed of the interplanetary shock driven by a solar flare. Cliver et al. (1990) proposed the following relationship between the maximum solar wind flow speed $\left(V_{\max }\right)$ and the shock transit speed $\left(V_{\mathrm{sh}}\right)$ :

$$
V_{\max }=0.775 V_{\mathrm{sh}}-40[\mathrm{~km} / \mathrm{s}] .
$$

Belov et al. (2008) also proposed a similar type of empirical relationship between $V_{\max }$ and $V_{\text {sh }}$ :

$$
V_{\max }=(220 \pm 6)+(0.52 \pm 0.03) V_{\mathrm{sh}}[\mathrm{km} / \mathrm{s}]
$$

Table 2 shows the event list of the solar flares with the corresponding values of $V_{\text {sh }}$ and $V_{\text {max }}$, which we estimated. The solar flares themselves are the same as those in Table 1. From the comparison between Table 2 and the estimated average solar wind speed of $960 \mathrm{~km} / \mathrm{s}$ in the previous section, the final solar flare in Table 2 is the most likely origin of the second SSC and the development of the main phase of the extreme storm. The occurrence of a type IV solar radio burst suggests that this solar flare was accompanied by CME. Further evidence is the solar wind flow speed at 22-23 UT on March 14 obtained from the OMNI database. During this period, the solar wind speed

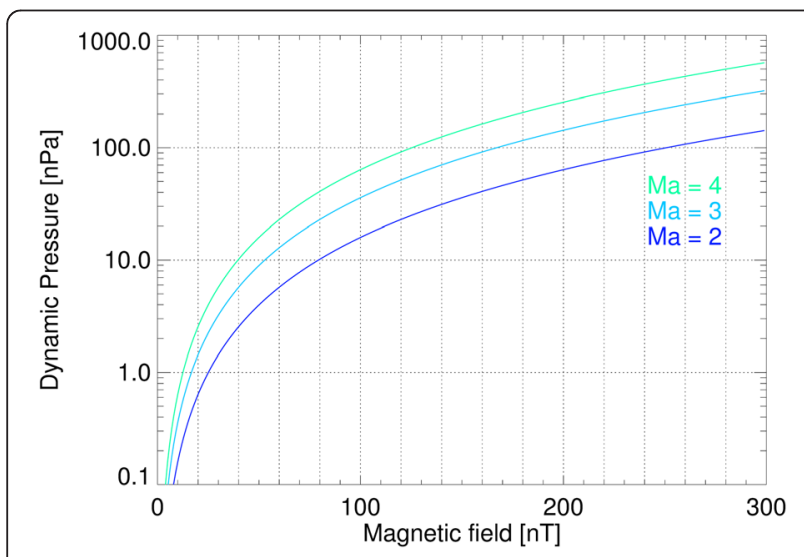

Fig. 3 Alfvén Mach number $\left(M_{A}\right)$ as a function of magnetic field intensity $[\mathrm{n} T]$ and dynamic pressure $[\mathrm{nPa}]$. The curves for $M_{\mathrm{A}}=2,3$, and 4 are plotted in different colors was about $800 \mathrm{~km} / \mathrm{s}$. Because no additional impulsive signature was recorded on the ground magnetometers after the second SSC, the flow at the end of March 14 appears to be a continuation of the high-speed stream originating from the interplanetary shock that produced the second SSC. The X1.3/2B solar flare on March 11 is a candidate for the origin of the second SSC because a possible halo CME corresponding to this flare was reported. However, the maximum solar wind speed estimated from this event was slower than that estimated from our study. On the basis of these results, the origin of the second SSC of this storm event appears to be the M7.8/2B solar flare that began at 0016 UT on March 12, 1989. Duration of this solar flare is $29 \mathrm{~min}$. The X1.3/2B solar flare on March 11 could be another candidate for the origin of the second SSC because of the ambiguity of the solar wind speed estimation.

Previous studies have suggested that solar flares of modest intensity and duration can produce severe geomagnetic storms. In the case of the solar sources of the Bastille geomagnetic storm that occurred on July 15-17, 2000, with a Dst minimum of $-301 \mathrm{nT}$, the duration of the solar flare was $40 \mathrm{~min}$ (Andrews 2001). In case of the solar sources of the Halloween geomagnetic storm that occurred November 20-21, 2003, with a Dst minimum of $-422 \mathrm{nT}$, three longdurational event (LDE) flares with intensities less than M5.0 were reported (e.g., Gopalswamy et al. 2005). This demonstrates that a strong geoeffective solar wind driver can be produced from a solar flare modest of intensity and duration.

The dynamic pressure of the solar wind in this event may have been extreme because the GMC occurred around the noon sector and in the dawn and dusk sectors. This indicates that extreme dynamic pressure enhancement may have compressed the magnetosphere. However, it is unclear whether we can apply the empirical formula for the magnetopause location to such an extreme condition, especially for the dawn and dusk sectors, because the static gas pressure may have some effect on the magnetopause location, which is not considered in this empirical formula. By simply applying the empirical formula for the dawn and dusk sectors of the GMC on March 13-14, 1989, the solar wind dynamic pressure was 


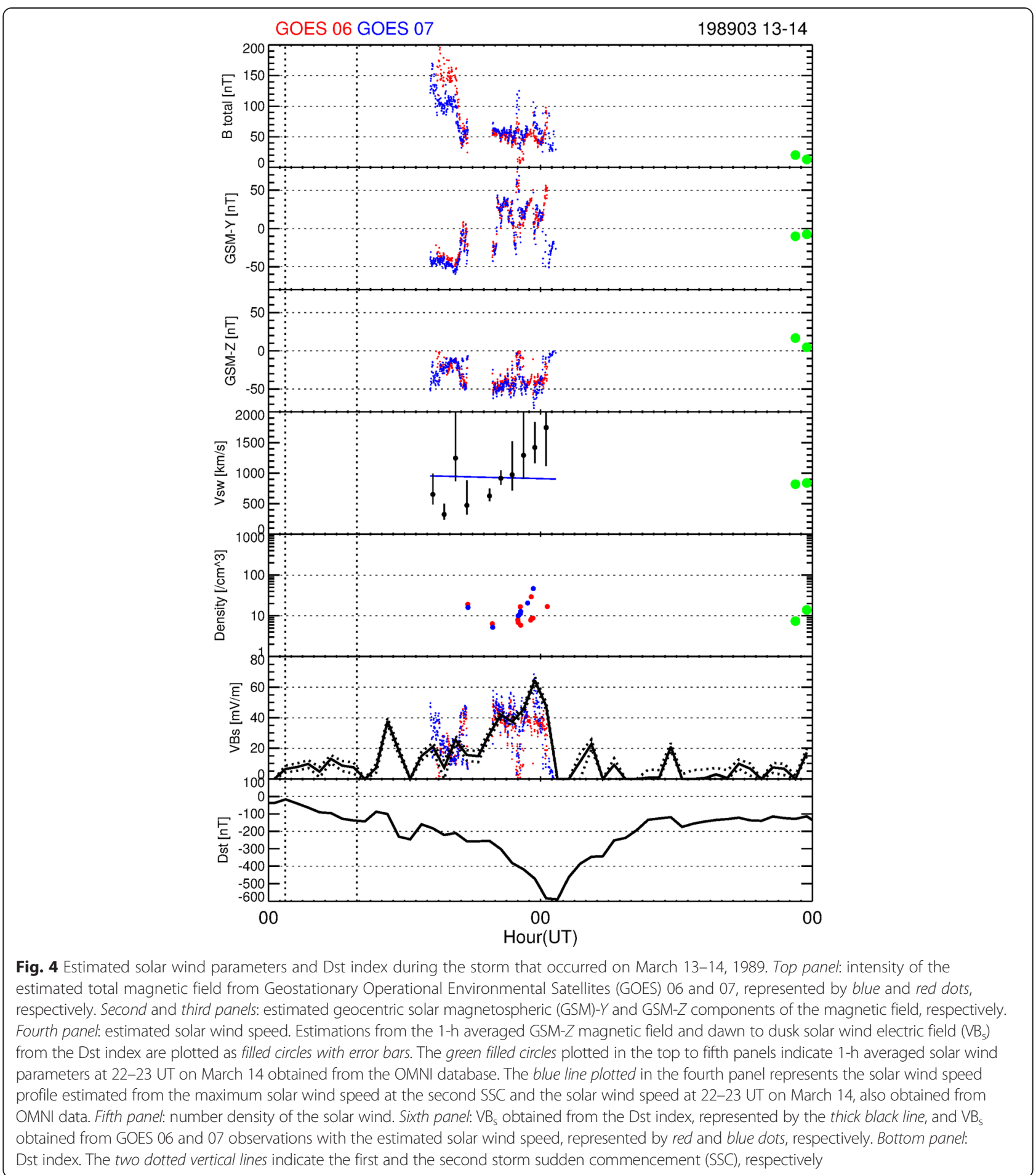

estimated to be more than $100 \mathrm{nPa}$. However, there is insufficient information to confirm such an extreme dynamic pressure of the solar wind. This point should be examined in detail for future extreme storm events by considering a variety of data.

Our estimations for the GSM- $Y$ and GSM- $Z$ components of the IMF strongly depended on the assumption of
$M_{\mathrm{A}}>2$. Figure 3 shows the $M_{\mathrm{A}}$ as a function of the magnetic field intensity and dynamic pressure for low values of $M_{\mathrm{A}}$. The curves for $M_{\mathrm{A}}=2,3$, and 4 are plotted in different colors. The condition $M_{\mathrm{A}}>2$ is mostly satisfied in the dynamic pressure range between 10 and $100 \mathrm{nPa}$ and the magnetic field intensity range between 50 and $100 \mathrm{nT}$. In contrast, $M_{\mathrm{A}}<2$ is mostly satisfied in the 


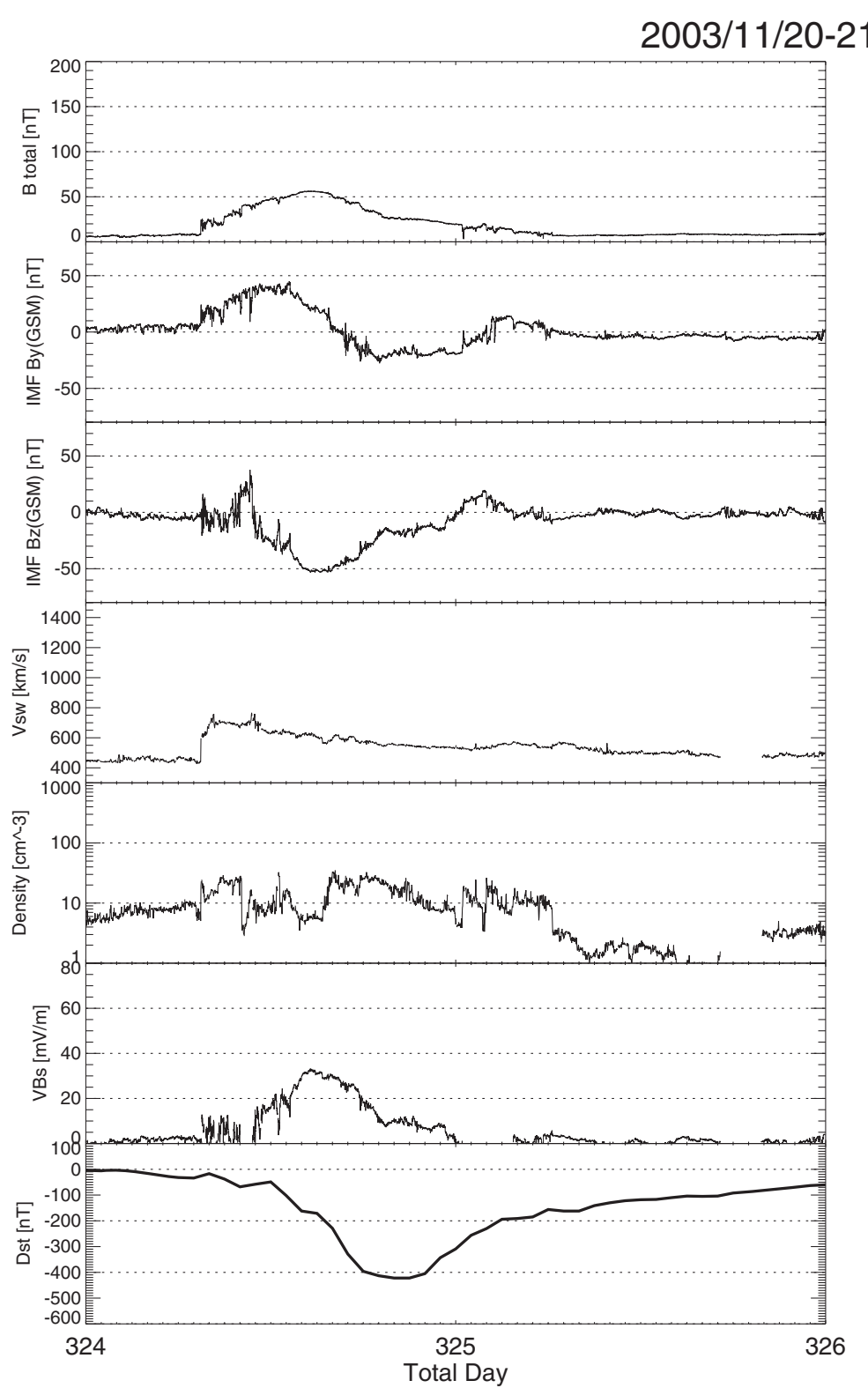

Fig. 5 Solar wind parameters obtained from the ACE satellite and the Dst index during the storm that occurred on November 20-21, 2003. Top panel: intensity of the magnetic field. Second panel: geocentric solar magnetospheric (GSM)-Y component of the magnetic field. Third panel: GSM-Z component of the magnetic field. Fourth panel: solar wind speed. Fifth panel: number density of the solar wind. Sixth panel: dawn to dusk solar wind electric field (VBs). Bottom panel: Dst index

dynamic pressure range between 10 and $100 \mathrm{nPa}$ and the magnetic field intensity range between 200 and $300 \mathrm{nT}$. This means that GOES satellite observation cannot distinguish between both upstream conditions of the magnetic field because in the former case, it is compressed fourfold and in the latter case, there is no compression. Therefore, GOES observed similar magnetic fields from both solar wind conditions. Thus, we cannot deny the possibility of another extreme state of solar wind. If $M_{\mathrm{A}}<2$ during the GMC observed by GOES, then a CME with a GSM- $Z$ component of the IMF of less than $-200 \mathrm{nT}$ reached Earth because there was no compression of the IMF. If a linear relationship between the $\mathrm{VB}_{\mathrm{s}}$ and the injection rate of the ring current (the Dst index) is applied, the solar wind speed should be $250 \mathrm{~km} / \mathrm{s}$. This condition is quite slow and unusual in magnitude and therefore provides an ineffective explanation for the rapid decline of the high-speed solar wind stream that caused the second SSC. Moreover, there is no known solar origin of a solar wind with this speed. On the basis of this discussion, therefore, the condition $M_{\mathrm{A}}>2$ was adopted for our data analysis. 
A summary of the estimated solar wind parameters is shown in Fig. 4. The green filled circles plotted in the top to fifth panels indicate $1-\mathrm{h}$ averaged solar wind parameters at 22-23 UT on March 14 obtained from the OMNI database. The blue line plotted in the fourth panel from the top is the solar wind speed profile estimated from the maximum solar wind speed at the second SSC and the solar wind speed at 22-23 UT at March 14 also obtained from OMNI data. The estimated solar wind conditions for the storm that occurred on March 13-14, 1989, were compared with those for the November 20-21, 2003, storm, which was the fifth largest since 1957; its Dst minimum was $-422 \mathrm{nT}$. This event is also one of the best extreme events in terms of direct continuous solar wind data recorded during the entire storm. Figure 5 shows the solar wind parameters obtained from the ACE satellite and the Dst index during the storm that occurred on November 20-21. The maximum amplitude of the GSM- $Z$ component of the IMF is comparable for both events at about $-50 \mathrm{nT}$, but the 5 -h duration of the intense southward IMF for the March 1989 event is longer than that for the November 2003 event at $2.5 \mathrm{~h}$. Another difference is the solar wind flow speed, which at $960 \mathrm{~km} / \mathrm{s}$ during the development of the strong ring current in the March 1989 event was 1.4 times faster than that in the November 2003 event at $700 \mathrm{~km} / \mathrm{s}$. The lower limit density of the solar wind for the March 1989 event, at $8-60 / \mathrm{cm}^{-3}$, may be comparable to that of the November 2003 event at $6-30 / \mathrm{cm}^{-3}$. A comparison of both extreme storm events suggests that the solar wind conditions that occurred during the storm on March 13-14, 1989, could have occurred more frequently than previously thought.

Our method of data analysis can be applied to severe geomagnetic storm events with no solar wind information. This type of reconstruction of solar wind information will be important for studying extreme geomagnetic storms in the past.

\section{Conclusions}

By using GOES magnetic field data and the Dst index, we have examined the solar wind conditions during the extreme geomagnetic storm that occurred on March 13-14, 1989. The solar flare possibly associated with the second SSC of this storm event was identified as the March $12 \mathrm{M} 7.3 / 2 \mathrm{~B}$ flare. During the $5 \mathrm{~h}$ in which the main phase of the storm rapidly developed, IMF $B_{\mathrm{z}}$ was estimated to be about $-50 \mathrm{nT}$ with a solar wind speed of about $960 \mathrm{~km} / \mathrm{s}$, assuming an $M_{\mathrm{A}}$ during this period of more than 2 . The number density of the solar wind during the GMC was at least $8-60 / \mathrm{cm}^{-3}$. Although these solar wind conditions are similar to those for the storm that occurred on November 20-21, 2003, the duration of the southward IMF was longer and the solar wind speed was 1.5 times faster in the latter event. This means that events such as the geomagnetic storm that occurred on March 13-14 could have occurred more frequently than previously thought.

\section{Abbreviations}

ACE: advanced composition explorer; $M_{\mathrm{A}}$ : Alfvén Mach number; CMD: central meridian distance; CME: coronal mass ejection; $\mathrm{VB}_{\mathrm{s}}$ : dawn to dusk solar wind electric field; GSM: geocentric solar magnetosphere; GIC: geomagnetically induced current; GOES: Geostationary Operational Environmental Satellite; GMC: geosynchronous magnetopause crossing; HAFv.2: Hakamada-Akasofu-Fry version 2; IMF: interplanetary magnetic field; IMP: interplanetary monitoring platform; $E_{m}$ : merging electric field; SMM: Solar Maximum Mission; STEREO: Solar Terrestrial Relations Observatory; SSC: storm sudden commencement.

\section{Competing interests}

The authors declare that they have no competing interests.

\section{Authors' contributions}

TN proposed the topic and analyzed the data. RK and MK collaborated with the corresponding author in the construction of the manuscript. All authors read and approved the final manuscript.

\section{Authors' information}

TN is a research manager of the Space Environment and Informatics Laboratory, Applied Electromagnetic Research Institute, NICT. RK is an associate professor of the National Institute of Polar Research, and SOKENDAI (the Graduate University for Advanced Studies). MK is a senior researcher of the Space Environment and Informatics Laboratory, Applied Electromagnetic Research Institute, NICT.

\section{Acknowledgments}

We thank the National Oceanic and Atmospheric Administration and the World Data Center for Geomagentism, Kyoto, for providing GOES magnetometer data and the Dst index, respectively. We also thank the ACE instrument teams (SWEPAM and MAG) and the ACE Science Center for providing the ACE data. The OMNI data were obtained from the GSFC/SPDF OMNIWeb interface at http://omniweb.gsfc.nasa.gov.

\section{Author details}

${ }^{1}$ National Institute of Information and Communications Technology, Tokyo, Japan. ${ }^{2}$ National Institute of Polar Research, Tokyo, Japan. ${ }^{3}$ SOKENDAI (The Graduate University for Advanced Studies), Tokyo, Japan.

Received: 31 October 2014 Accepted: 15 May 2015

Published online: 27 May 2015

\section{References}

Allen J, Sauer H, Frank L, Reiff P (1989) Effects of the March 1989 solar activity. Eos Trans AGU 70(46):1479-1488. doi:10.1029/89EO00409

Andrews MD (2001) LASCO and EIT observations of the Bastille Day 2000 solar storm. Solar Phys 204:179-196

Baker DN, Li X, Pulkkinen A, Ngwira CM, Mays ML, Galvin AB, Simunac KDC (2013) A major solar eruptive event in July 2012: defining extreme space weather scenarios. Space Weather 11:585-591. doi:10.1002/swe.20097

Batista IS, De Paula ER, Abdu MA, Trivedi NB, Greenspan ME (1991) Ionospheric effects of the March 13, 1989, magnetic storm at low and equatorial latitudes. J Geophys Res 96(A8):13943-13952. doi:10.1029/91JA01263

Belov AV, Eroshenko EA, Oleneva VA, Yanke VG (2008) Connection of Forbush effects to the X-ray flares. J Atomos Sol Terr Phys 70:342-350. doi:10.1016/j.jastp.2007.08.021

Burkepile JT, St. Cyr OC (1993) A revised and expanded catalogue of mass ejections observed by the solar maximum mission coronagraph. NCAR, TN-369+ STR

Cliver EW, Feynman J, Garrett HB (1990) An estimate of the maximum speed of the solar wind, 1938-1989. J Geophys Res 95(A10):17103-17112. doi:10.1029/JA095iA10p17103

Cliver EW, Balasubramaniam KS, Nitta NY, Li X (2009) Great geomagnetic storm of 9 November 1991: association with a disappearing solar filament. J Geophys Res 114:A00A20. doi:10.1092/2008JA013232 
Feynman J, Hundhausen AJ (1994) Coronal mass ejections and major solar flares: the great active center of March 1989. J Geophys Res 99(A5):8451-8464. doi:10.1029/94JA00202

Fry CD, Sun W, Deehr CS, Dryer M, Smith Z, Akasofu S-I, Tokumaru M, Kojima M (2001) Improvements to the HAF solar wind model for space weather predictions. J Geophys Res 106(A10):20985-21001. doi:10.1029/2000JA000220

Fry CD, Dryer M, Smith Z, Sun W, Deehr CS, Akasofu S-I (2003) Forecasting solar wind structures and shock arrival times using an ensemble of models. J Geophys Res 108(A2):1070. doi:10.1029/2002JA009474

Fujii R, Fukunishi H, Kokubun S, Sugiura M, Tohyama F, Hayakawa H, Tsuruda K, Okada T (1992) Field-aligned current signatures during the March 13-14, 1989, great magnetic storm. J Geophys Res 97(A7):10703-10715. doi:10.1029/92JA00171

Gopalswamy N, Yashiro S, Michalek G, Xie H, Lepping RP, Howard RA (2005) Solar source of the largest geomagnetic storm of cycle 23. Geophys Res Lett 32:L12S09. doi:10.1029/2004GL021639

Greenspan ME, Rasmussen CE, Burke WJ, Abdu MA (1991) Equatorial density depletions observed at $840 \mathrm{~km}$ during the great magnetic storm of March 1989. J Geophys Res 96(A8):13931-13942. doi:10.1029/91JA01264

Hajkowicz LA (1991) Global onset and propagation of large-scale travelling ionospheric disturbances as a result of the great storm of 13 March 1989. Planet Space Sci 39(4):583-593. doi:10.1016/0032-0633(91)90053-D

Kataoka R (2013) Probability of occurrence of extreme magnetic storms Space Weather 11:214-218. doi:10.1002/swe.20044

Lakshmi DR, Rao BCN, Jain AR, Goel MK, Reddy BM (1991) Response of equatorial and low latitude F-region to the great magnetic storm of 13 March 1989 (1991). Ann Geophys 9:286-290

Li X, Temerin M, Tsurutani BT, Alex S (2006) Modeling of 1-2 September 1859 super magnetic storm. Adv Space Res 38:273-279

McKenna-Lawlor SMP, Dryer M, Fry CD, Sun W, Lario D, Deehr CS, Sanahuja B, Afonin VA, Verigin MI, Kotova GA (2005) Predictions of energetic particle radiation in the close Martian environment. J Geophys Res 110(A3):A03102. doi:10.1029/2004JA010587

Nagatsuma T, Asai KT, Kataoka R, Hori T, Miyoshi Y (2007) S-M-I coupling during the super storm on 20-12 November 2003. Adv Geosci 14:237-244

O'Brien TP, McPherron RL (2000) An empirical phase space analysis of ring current dynamics: solar wind control of injection and decay. J Geophys Res 105(A4):7707-7719. doi:10.1029/1998JA000437

Okada T, Hayakawa H, Tsuruda K, Nishida A, Matsuoka A (1993) EXOS D observations of enhanced electric fields during the giant magnetic storm in March 1989. J Geophys Res 98(A9):15417-15424. doi:10.1029/93JA01128

Rasmussen CE, Greenspan ME (1993) Plasma transport in the equatorial ionosphere during the great magnetic storm of March 1989. J Geophys Res 98(A1):285-292. doi:10.1029/92JA02199

Rich FJ, Denig WF (1992) The major magnetic storm of March 13-14, 1989, and associated ionosphere effects. Can J Phys 70(7):510-525. doi:10.1139/p92-086

Rufanach CL, Martin RF Jr, Sauer HH (1989) A study of geosynchronous magnetopause crossings. J Geophys Res 94(A11):15125-15134 doi:10.1029/JA094iA11p151254

Shinbori A, Nishimura Y, Ono T, lizima M, Kumamoto A, Oya H (2005) Electrodynamics in the duskside inner magnetosphere and plasmasphere during a super magnetic storm on March 13-15, 1989. Earth Planets Space 57:643-659

Shue JH, Song P, Russell CT, Steinberg JT, Chao JK, Zastenker G, Vaisberg OL, Kokubun S, Singer HJ, Detman TR, Kawano H (1998) Magnetopause location under extreme solar wind conditions. J Geophys Res 103(A8):17691-17700. doi:10.1029/98JA01103

Shue JH, Song P, Russell CT, Chao JK, Yang YH (2000) Toward predicting the position of the magnetopause within geosynchronous orbit. J Geophys Res 105(A2):2641-2656. doi:10.1029/1999JA900467

Sojka JJ, Schunk RW, Denig WF (1994) Ionospheric response to the sustained high geomagnetic activity during the March ' 89 great storm. J Geophys Res 99(A11):21341-21352. doi:10.1029/94JA01765

Tanaka T (2007) Magnetosphere-ionosphere convection as a compound system Space Sci Rev. doi: 10.1007/s11214-007-9168-4

Walker GO, Wong YW (1993) lonospheric effects observed throughout East Asia of the large magnetic storm of 13-15 March 1989. J Atmos Terr Phys 55(7):995-1008. doi:10.1016/0021-9169(93)90093-E

Yeh KC, Lin KH, Conkright RO (1992) The global behavior of the March 1989 ionospheric storm. Can J Phys 70(7):532-543. doi:10.1139/p92-088

\section{Submit your manuscript to a SpringerOpen ${ }^{\odot}$ journal and benefit from:}

- Convenient online submission

- Rigorous peer review

- Immediate publication on acceptance

- Open access: articles freely available online

- High visibility within the field

- Retaining the copyright to your article

Submit your next manuscript at $>$ springeropen.com 